The Astrophysical Journal, 663:450-460, 2007 July 1

(C) 2007. The American Astronomical Society. All rights reserved. Printed in U.S.A.

\title{
THERMODYNAMIC DESCRIPTION OF INELASTIC COLLISIONS IN GENERAL RELATIVITY
}

\author{
Jörg Hennig and Gernot Neugebauer \\ Theoretisch-Physikalisches Institut, Friedrich-Schiller-Universität Jena, D-07743 Jena, Germany; j.hennig@tpi.uni-jena.de \\ AND \\ Marcus Ansorg \\ Max-Planck-Institut für Gravitationsphysik, Albert-Einstein-Institut, D-14476 Golm, Germany; marcus.ansorg@aei.mpg.de \\ Received 2007 January 29; accepted 2007 March 25
}

\begin{abstract}
We discuss head-on collisions of neutron stars and disks of dust ("galaxies") following the ideas of equilibrium thermodynamics, which compares equilibrium states and avoids the description of the dynamical transition processes between them. As an always present damping mechanism, gravitational emission results in final equilibrium states after the collision. In this paper we calculate selected final configurations from initial data of colliding stars and disks by making use of conservation laws and solving the Einstein equations. Comparing initial and final states, we can decide for which initial parameters two colliding neutron stars (nonrotating Fermi gas models) merge into a single neutron star and two rigidly rotating disks form again a final (differentially rotating) disk of dust. For the neutron star collision we find a maximal energy loss due to outgoing gravitational radiation of $2.3 \%$ of the initial mass, while the corresponding efficiency for colliding disks has the much larger limit of $23.8 \%$.
\end{abstract}

Subject headings: equation of state — galaxies: general — gravitation — gravitational waves — stars: neutron

\section{INTRODUCTION}

Collisions of compact objects are an important source of gravitational radiation. Much effort has recently been made to develop numerical methods and codes describing and simulating the underlying hydrodynamical and gravitational phenomena. After the pioneering work on numerical black hole evolutions by Eppley and Smarr in the 1970s (see, e.g., Eppley 1975; Smarr et al. 1976), head-on collisions were reinvestigated in the 1990s (Anninos et al. 1993, 1995; Anninos \& Brandt 1998) with good agreement between numerical and perturbation theoretical results. Long-term stable evolutions of black hole and neutron star collisions were successfully performed in the last 2 years (Sperhake et al. 2005; Fiske et al. 2005; Zlochower et al. 2005; Sperhake 2006; Löffler et al. 2006).

From a mathematical point of view collision processes are typical examples for initial boundary problems. In particular, we discuss head-on collisions of spheres and disks ${ }^{1}$ (see Fig. 1). Starting with bodies separated by a large ("infinite") distance, we may model the initial situation by a quasi-equilibrium configuration of two isolated bodies. Corresponding solutions for spheres and (rigidly rotating) disks can be found in the literature (see, e.g., Misner et al. 2002; Shapiro \& Teukolsky 1983; Neugebauer $\&$ Meinel 1993, 1994, 1995). The dynamical phase of the collision process is always accompanied by gravitational radiation. This damping mechanism results again in the formation of an equilibrium configuration after the collision. The rigorous mathematical description of the dynamical transition phase is difficult and requires extensive numerical investigations. However, interesting information about the collision can be obtained by comparing the initial and final (equilibrium) states. This thermodynamic idea avoids the analysis of the transition process and reduces the mathematical effort to solving the Einstein equations for the end products, which are stationary and axisymmetric in our case. The

\footnotetext{
${ }^{1}$ Disklike matter configurations play an important role in astrophysics, e.g., as models for galaxies, accretion disks, or intermediate phases in the merger process of two neutron stars.
}

solution makes use of conservation laws that transfer data extracted from the initial configurations (spheres and disks before the collision) to the final configurations.

While the initial configurations are available, the calculation of the final states is rather difficult. To cope with this problem for head-on colliding stars and disks, we make use of two heuristic principles:

1. Perfect fluid configurations at rest are spherically symmetric. Hence, the end product of two head-on colliding spheres without angular momentum is again a sphere (a fluid ball or a Schwarzschild black hole).

2. Dust configurations are two-dimensional ("extremely flattened") and axisymmetric (with nonvanishing angular momentum). Consequently, the dust matter after a head-on collision of two disks of dust is again two-dimensional and axisymmetric (a compact disk, a disk surrounded by dust rings, or a black hole surrounded by dust rings).

Although plausible, these principles have not been proved rigorously so far. ${ }^{2}$ For proofs under special assumptions see Beig \& Simon (1992) and Lindblom \& Masood-Ul-Alam (1994).

As illustrated in Figure 1, we confine ourselves to two problems: (1) head-on collisions of two identical spheres (stars) merging into a single fluid ball, and (2) head-on collisions of two identical disks of dust (galaxies) merging into a single disk. We will be able to formulate necessary conditions for the formation of these balls or disks. Obviously, the conditions will restrict the parameters of the initial configuration; a violation of the conditions would necessarily lead to other final states such as black holes or central disks surrounded by rings. To express the parameters of the final configuration in terms of the admissible initial parameters (the first goal of this paper), we have to solve the Einstein equations (numerically but) rigorously and to make use of the conservation laws for baryonic mass and angular momentum. There is no obstacle to an extension of the method. One could start a systematic investigation of other possible final states

\footnotetext{
2 In this context we refer to a new approach by Masood-Ul-Alam (2007).
} 
a)

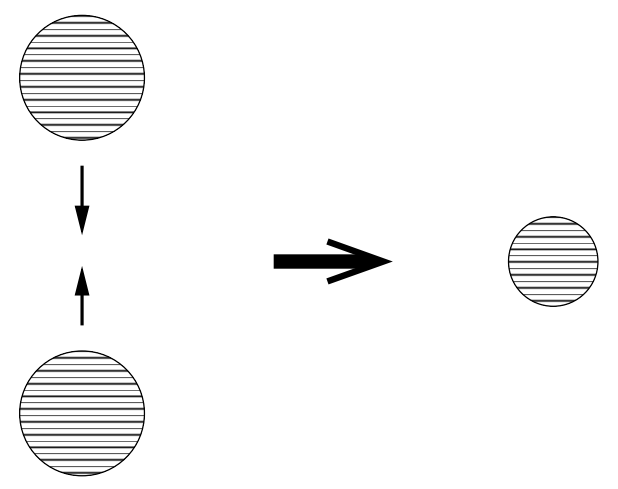

b)

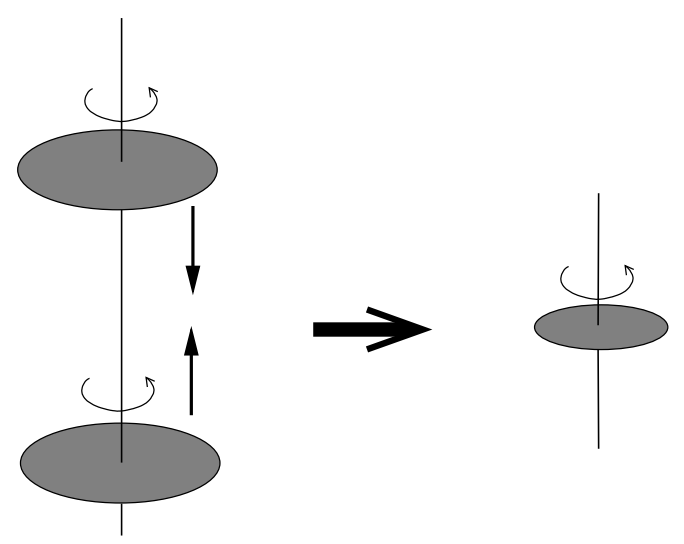

FIG. 1.-Model: collisions of $(a)$ two spherically symmetric stars; $(b)$ two rigidly rotating disks of dust.

after the collision (black holes, black holes with rings, etc.) making use of symmetries, conservation laws, and the heuristic principles 1 and 2 . An important point of the procedure would be the stability analysis of the end products. As for our investigation, there is important evidence from Newtonian gravity that rigidly or differentially rotating disks of dust are unstable. Nevertheless, we can expect that "stabilizing" effects (pressure due to internal kinetic energy) do not falsify our other goal: to estimate the maximal contribution of gravitational radiation to the total energy loss $\Delta M$. In general, the total energy loss calculated via the comparison of initial and final equilibrium configurations is only an upper limit for the energy loss (efficiency) due to gravitational emission. (It includes energy loss due to nongravitative radiation or mass ejection during the collision.) We present a disk collision model that is exclusively damped by gravitational radiation. The resulting differentially rotating disk is compared with a rigidly rotating disk of the same baryonic mass and angular momentum formed from the same initial disks under the additional influence of dissipative processes in the matter (see $\S 3.2$ ). Thus, we can compare the efficiencies of the two forms of dissipation.

In $\S 2$ we discuss, as introductory examples, the merger of two Schwarzschild stars and the collision of two (Fermi gas) neutron stars. Section 3 contains the main part of this paper, which is dedicated to the investigation of disk collisions. These discussions are based on a novel solution of the Einstein equations describing the final configuration. Here we continue the analysis of a previous paper (Hennig \& Neugebauer 2006), in which we discussed the collisions of rigidly rotating disks of dust with parallel (or antiparallel) angular momenta under the simplifying assumption that the final disk be again a rigidly rotating (or rigidly counterrotating) disk of dust. This assumption can only be justified if friction processes between the disk rings provide for a constant angular velocity throughout the disk. This model seems to be somewhat artificial and unsuited to determining the contribution of gravitational radiation to the total energy loss. Interestingly, our present investigation shows that the frictional contribution to the total energy loss for colliding rigidly rotating disks is comparably small.

\section{STAR COLLISIONS}

\subsection{Introductory Example: Schwarzschild Stars}

In order to demonstrate the method, we study the collision of two Schwarzschild stars, i.e., spherically symmetric perfect fluid stars with a constant mass density, $\mu=$ constant. Although not very realistic, this model illustrates the main steps of the method.
The matter of a Schwarzschild star is described by the perfect fluid energy momentum tensor

$$
T^{i j}=(\mu+p) u^{i} u^{j}+p g^{i j}
$$

with the pressure

$$
p(r)=\frac{\sqrt{1-(8 \pi \mu / 3) r^{2}}-\sqrt{1-(8 \pi \mu / 3) r_{0}^{2}}}{3 \sqrt{1-(8 \pi \mu / 3) r_{0}^{2}}-\sqrt{1-(8 \pi \mu / 3) r^{2}}} \mu,
$$

where $u^{i}, r$, and $r_{0}$ are the four-velocity, the radial coordinate, and the coordinate radius of the star, respectively. The interior Schwarzschild metric can be written as

$$
\begin{aligned}
d s^{2}= & \frac{d r^{2}}{1-(8 \pi \mu / 3) r^{2}}+r^{2}\left(d \vartheta^{2}+\sin ^{2} \vartheta d \varphi^{2}\right) \\
& -\left(\frac{3}{2} \sqrt{1-\frac{8 \pi \mu}{3} r_{0}^{2}}-\frac{1}{2} \sqrt{1-\frac{8 \pi \mu}{3} r^{2}}\right) d t^{2},
\end{aligned}
$$

and the exterior Schwarzschild solution is

$$
d s^{2}=\frac{d r^{2}}{1-2 M / r}+r^{2}\left(d \vartheta^{2}+\sin ^{2} \vartheta d \varphi^{2}\right)-\left(1-\frac{2 M}{r}\right) d t^{2} .
$$

Note that we use the normalized units where $c=1$ for the speed of light and $G=1$ for Newton's gravitational constant.

The gravitational mass $M$,

$$
M=\frac{4 \pi \mu}{3} r_{0}^{3},
$$

follows from the matching condition at the star's surface, and the baryonic mass $M_{0}$ is given by

$M_{0}=\int_{t=t_{0}} \mu u^{t} \sqrt{-g} d r d \vartheta d \varphi=4 \pi \mu \int_{0}^{r_{0}} \frac{r^{2} d r}{\sqrt{1-(8 \pi \mu / 3) r^{2}}}$.

Now we apply these formulae to the head-on collision of two stars. Restricting ourselves to collisions of two identical Schwarzschild stars, we assume that again the final star is a Schwarzschild star 
and has the same mass density (e.g., nuclear matter density),

$$
\tilde{\mu}=\mu
$$

where from now on tildes denote quantities after the collision.

The conservation of baryonic mass during the collision process

$$
\tilde{M}_{0}=2 M_{0}
$$

allows one to calculate the parameters of the final star as a function of the initial parameters. With equations (5), (6), and (7) the conservation equation (8) can be written as

$$
\begin{array}{r}
\arcsin \left(\sqrt{\frac{2 M}{r_{0}}} \frac{\tilde{r}_{0}}{r_{0}}\right)-\sqrt{\frac{2 M}{r_{0}}} \frac{\tilde{r}_{0}}{r_{0}} \sqrt{1-\frac{2 M}{r_{0}} \frac{\tilde{r}_{0}^{2}}{r_{0}^{2}}} \\
=2\left(\arcsin \sqrt{\frac{2 M}{r_{0}}}-\sqrt{\frac{2 M}{r_{0}}} \sqrt{1-\frac{2 M}{r_{0}}}\right) ;
\end{array}
$$

i.e., the radius ratio $\tilde{r}_{0} / r_{0}$ is a function of the initial mass-radius ratio $2 M / r_{0}$. Hence, we may express the efficiency $\eta$ of conversion of mass into gravitational radiation,

$$
\eta=1-\frac{\tilde{M}}{2 M}=1-\frac{1}{2}\left(\frac{\tilde{r}_{0}}{r_{0}}\right)^{3}
$$

and the mass-radius ratio of the final star,

$$
\frac{2 \tilde{M}}{\tilde{r}_{0}}=\frac{2 M}{r_{0}}\left(\frac{\tilde{r}_{0}}{r_{0}}\right)^{2}
$$

in terms of $2 M / r_{0}$.

The resulting parameter relations are plotted in Figure 2. For Schwarzschild stars the coordinate radius is restricted by the Buchdahl condition, i.e.,

$$
r_{0}>\frac{9}{8}(2 M), \quad \tilde{r}_{0}>\frac{9}{8}(2 \tilde{M}) .
$$

As a consequence, the first plot shows that "relativistic" initial stars with $2 M / r_{0}>0.6482$. . can never merge into a new Schwarzschild star with the same mass density $\mu$. The "physical" parts of the parameter relations are shown as solid lines, while the forbidden parts are dashed. According to the bottom panel, the efficiency $\eta$ cannot exceed a maximal value of $\eta_{\max } \approx 19.7 \%$.

\subsection{Neutron Stars: Completely Degenerate Ideal Fermi Gas}

In order to extend the discussion of the previous section to a more realistic star model, we replace the equation of state $\mu=$ constant by the equation for a completely degenerate ideal Fermi gas of neutrons.

The (interior) line element of a spherically symmetric star can be written as

$$
d s^{2}=e^{2 \lambda(r)} d r^{2}+r^{2}\left(d \vartheta^{2}+\sin ^{2} \vartheta d \varphi^{2}\right)-e^{2 \nu(r)} d t^{2},
$$
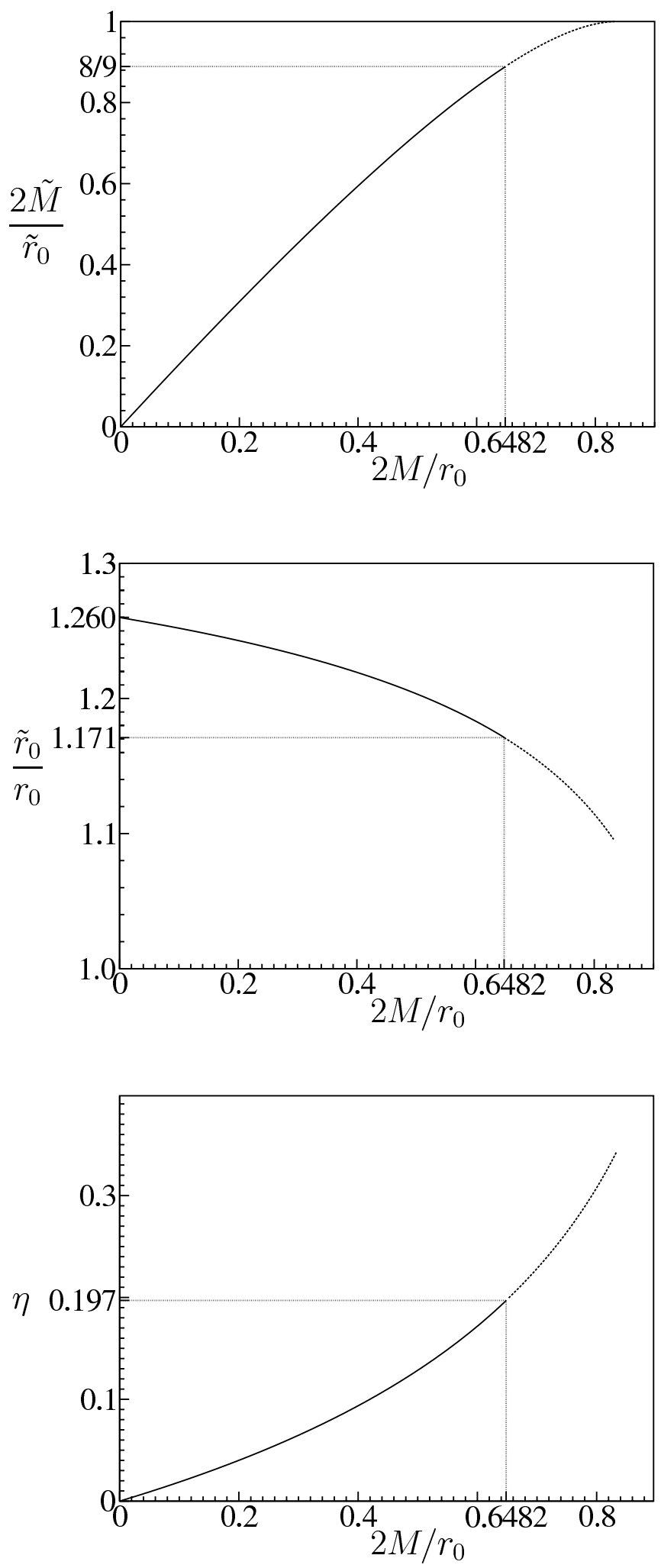

FIG. 2.-Parameter relations for colliding Schwarzschild stars. The final mass-radius ratio $2 \tilde{M} / \tilde{r}_{0}$, the radius ratio $\tilde{r}_{0} / \tilde{r}$, and the efficiency $\eta$ are plotted as functions of the initial mass-radius ratio $2 M / r_{0}$. Dashed parts of the lines mark regions inaccessible due to the Buchdahl inequality $2 \tilde{M} / \tilde{r}_{0}<\frac{8}{9}$.

and the matter is again described by the perfect fluid energy momentum tensor

$$
T^{i j}=(\mu+p) u^{i} u^{j}+p g^{i j} .
$$



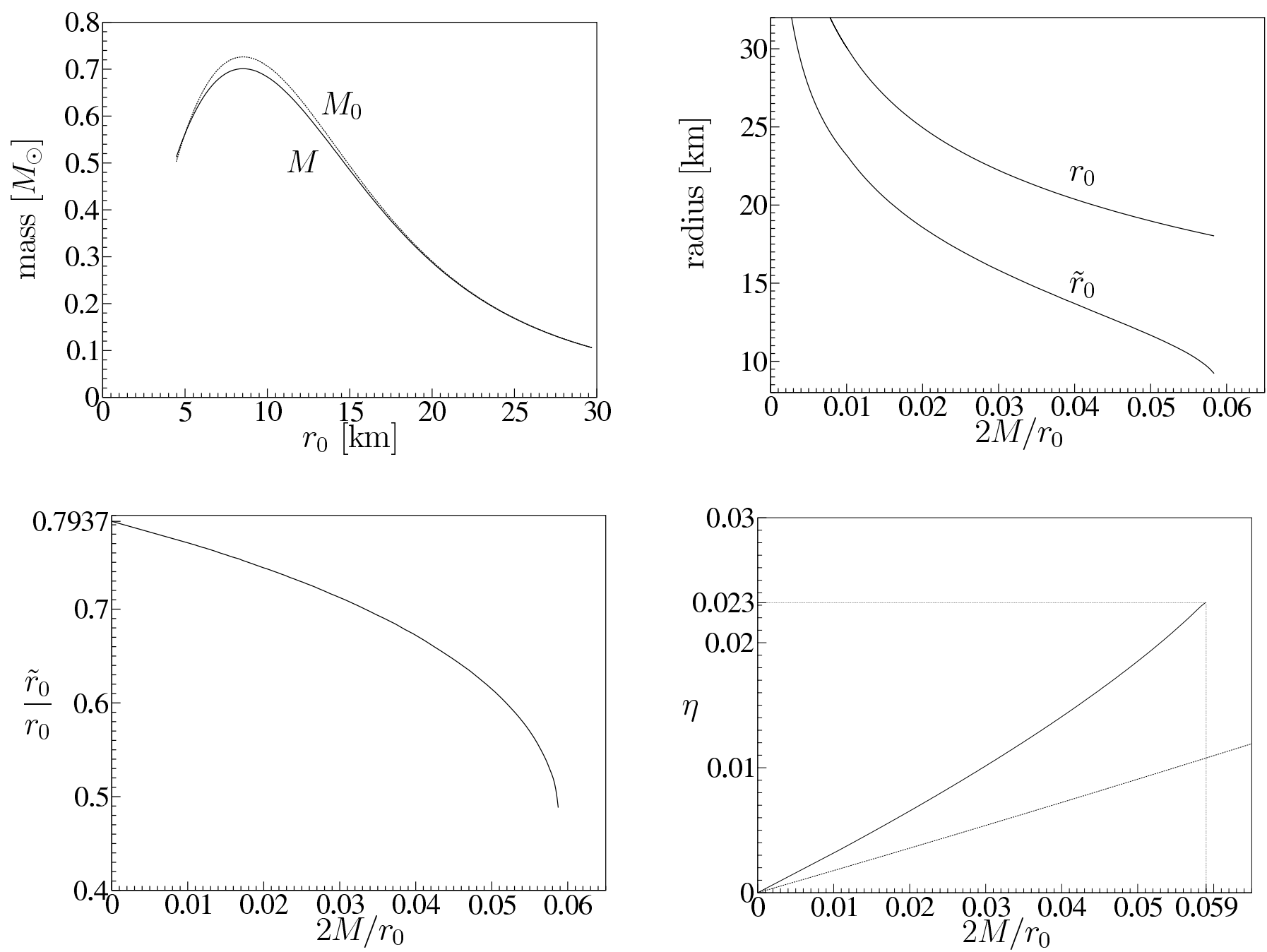

FIG. 3.- Parameter relations for the collisions of neutron stars made up of degenerate neutrons (see eq. [19]). Top left: Mass-radius relations for the baryonic mass $M_{0}$

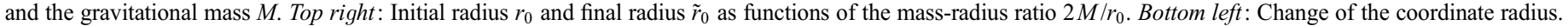
Bottom right: Efficiency $\eta$ compared to the efficiency of the collision of Schwarzschild stars (dashed line).

With the definition of a new metric function $m(r)$ by

$$
e^{2 \lambda(r)}=\frac{1}{1-[2 m(r) / r]}
$$

the field equations can be written in the TOV form (see, e.g., Shapiro \& Teukolsky 1983),

$$
\begin{gathered}
\frac{d m}{d r}=4 \pi r^{2} \mu, \quad m(0)=0 \\
\frac{d p}{d r}=-\frac{m}{r^{2}} \mu\left(1+\frac{p}{\mu}\right)\left(1+\frac{4 \pi r^{3} p}{m}\right)\left(1-\frac{2 m}{r}\right)^{-1}, \quad p(0)=p_{c} \\
\frac{d \nu}{d r}=-\frac{1}{\mu} \frac{d p}{d r}\left(1+\frac{p}{\mu}\right)^{-1}
\end{gathered}
$$

where $p_{c}$ is the pressure in the center of the star.

We solve these equations for the completely degenerate ideal Fermi gas of neutrons with the equation of state (see, e.g., Shapiro \& Teukolsky 1983)

$$
p=c_{1} f(x), \quad \rho=c_{2} x^{3}, \quad \mu=\rho+c_{1} g(x),
$$

where

$$
\begin{gathered}
f(x)=x\left(2 x^{2}-3\right) \sqrt{1+x^{2}}+3 \ln \left(x+\sqrt{1+x^{2}}\right), \\
g(x)=8 x^{3}\left(\sqrt{1+x^{2}}-1\right)-f(x), \\
c_{1}=\frac{\pi m_{n}^{4}}{3 h^{3}}, \quad c_{2}=\frac{8 \pi m_{n}^{4}}{3 h^{3}}
\end{gathered}
$$

with the neutron mass $m_{n}=1.6749286 \times 10^{27} \mathrm{~kg}$ and Planck's constant $h=6.626076 \times 10^{-34} \mathrm{~J}$ s. By solving the TOV equations (16) and (17) with the equation of state given by equation (19) for a sequence of values of the central density, one can calculate the corresponding radii of the stars as the first zero $r_{0}$ of $p(r)$, their gravitational mass from $M=m\left(r_{0}\right)$, and their baryonic mass as

$$
M_{0}=4 \pi \int_{0}^{r_{0}} \frac{\rho(r) r^{2} d r}{\sqrt{1-2 m(r) / r}} .
$$

The resulting mass-radius relations are shown in the top left panel of Figure 3. 
Again the baryonic mass is an invariant of the collision, i.e.,

$$
\tilde{M}_{0}=2 M_{0}
$$

for the collision of two identical initial stars. This equation has to be analyzed together with the mass-radius relations. [Thereby we take into account only stars in the monotonic decreasing part of the mass-radius relation $M_{0}\left(r_{0}\right)$.] The resulting parameter relations are shown in the remaining panels of Figure 3. For the maximum of the efficiency one finds $\eta_{\max } \approx 2.3 \%$, i.e., a comparably small value in view of the maximal efficiency $\eta_{\max } \approx 19.7 \%$ for collisions of Schwarzschild stars. The reason is the relatively small maximal mass of $M_{\max } \approx 0.7 M_{\odot}$ permitted by the equation of state given by equation (19) that excludes highly relativistic values for the mass-radius ratio $2 M / r_{0}$. However, compared to Schwarzschild stars with the same parameter $2 M / r_{0}$, the collisions of neutron stars are more efficient (see the bottom right panel of Fig. 3).

Another difference is the change of the coordinate radii. While two Schwarzschild stars merge into a Schwarzschild star with a coordinate radius bigger than the initial radius, $\tilde{r}_{0} / r_{0}>1$ (see Fig. 2), the resulting neutron star is smaller than the initial neutron stars, $\tilde{r}_{0} / r_{0}<1$ (see Fig. 3).

\section{DISK COLLISIONS}

Collisions of disks of dust require more effort. In particular, the discussion of the final equilibrium state is based on a solution of a free boundary value problem to the Einstein equations. At the first glance, the conservation laws for baryonic mass and angular momentum are not sufficient to formulate a complete set of boundary conditions for the configuration after the collision. However, excluding nongravitational dissipation, we may replace the global conservation laws, as used in $\S 2$, by local ones. Due to the geodesic motion of dust particles, the baryonic mass and the angular momentum of each of the rings forming the disk are conserved separately (see Fig. 5 below). Using such local conservation laws, we are able to solve (numerically) the boundary value problem for the final state after the head-on collision of two aligned rigidly rotating disks of dust with parallel angular momenta (see Fig. 1).

\subsection{Initial Disks: Rigidly Rotating Disks of Dust}

The free boundary value problem for the relativistic rigidly rotating disk of dust (RR disk) was discussed by Bardeen \& Wagoner $(1969,1971)$ using approximation methods and analytically solved in terms of ultraelliptic theta functions by Neugebauer \& Meinel $(1993,1994,1995)$ using the inverse scattering method. The line element of the stationary (Killing vector: $\xi^{i}$ ) and axisymmetric (Killing vector: $\eta^{i}$ ) spacetime may be written in the Weyl-Lewis-Papapetrou standard form

$$
\begin{gathered}
d s^{2}=e^{-2 U}\left[e^{2 k}\left(d \rho^{2}+d \zeta^{2}\right)+\rho^{2} d \varphi^{2}\right]-e^{2 U}(d t+a d \varphi)^{2} \\
\xi^{i}=\delta_{t}^{i}, \quad \eta^{i}=\delta_{\varphi}^{i}
\end{gathered}
$$

where the metric potentials $U=U(\rho, \zeta), k=k(\rho, \zeta)$, and $a=$ $a(\rho, \zeta)$ are given in terms of ultraelliptic theta functions.

The matter of the disk of dust is described by the energy momentum tensor

$$
T^{i j}=\varepsilon(\rho) \delta(\zeta) u^{i} u^{j}
$$

where $\varepsilon(\rho) \delta(\zeta)$ is the mass density with $\delta(\zeta)$ as Dirac's $\delta$-distribution. Due to the symmetries, the four-velocity of the dust particles is a linear combination of the two killing vectors,

$$
u^{i}=e^{-V_{0}}\left(\xi^{i}+\Omega_{0} \eta^{i}\right), \quad u^{i} u_{i}=-1,
$$

whence

$$
\left(\xi^{i}+\Omega_{0} \eta^{i}\right)\left(\xi_{i}+\Omega_{0} \eta_{i}\right)=-e^{2 V_{0}},
$$

where $\Omega_{0}$ is the angular velocity of the particles forming the disk and $V_{0}$ is a redshift parameter. Rigid rotation means $\Omega_{0}=$ constant in the disk. Since dust particles move geodesically, this assumption implies $V_{0}=$ constant in the disk. Hence, the boundary condition given by equation (28) and as a consequence the RR disk solution contain two constant parameters. Alternatively to $\Omega_{0}$ and $V_{0}$, one may choose the coordinate radius $\rho_{0}$ of the disk and a centrifugal parameter $\mu=2 \Omega_{0}^{2} \rho_{0}^{2} e^{-2 V_{0}}(\mu \rightarrow 0$ turns out to be the Newtonian limit and $\mu \rightarrow 4.62966$. . . the ultrarelativistic limit, where the disk approaches the extreme Kerr black hole; for these and further properties see Neugebauer \& Meinel 1994; Neugebauer et al. 1996).

\subsection{Final Disk: Differentially Rotating Disk of Dust}

In a previous paper (Hennig \& Neugebauer 2006) we discussed head-on collisions of two (identical) rigidly rotating disks of dust merging into one rigidly rotating disk of dust. The model excluded mass ejection and made use of the conservation of baryonic mass and angular momentum (axisymmetry). From a thermodynamic point of view rigid rotation of the final disk means thermodynamic equilibrium, which is a result of dissipative processes during the dynamical phase. Hence, the energy difference between the initial state (two separated disks) and the final state (one rigidly rotating disk) is influenced by irreversible processes in the matter and outgoing electromagnetic radiation, as well as by emission of gravitational waves. The intention of this paper is to compare the contribution of these two effects by calculating the end product of a purely gravitational collision process, which we expect to be a differentially rotating disk of dust. Note that our thermodynamic analysis enables us to formulate necessary conditions for the parameters of the initial disks $(\mu$ restricted) to permit the formation of a final disk. To obtain sufficient conditions, one would have to solve the Einstein equations for the time-dependent transition phase, which is outside the scope of this paper.

In the next subsection we give a brief summary of the previous paper. After that, we see that the local conservation of baryonic mass and angular momentum is sufficient to calculate the final differentially rotating disk (numerically). Differentially rotating disks with arbitrary rotation law have already been studied (Ansorg \& Meinel 2000; Ansorg 2001). The point made here is that we are able to formulate a physically motivated rotation law as a result of a collision process.

\subsubsection{Formation of Rigidly Rotating Disks}

For the formation of an RR disk from two colliding RR disks under the influence of friction processes the conservation equations for baryonic mass and angular momentum,

$$
\tilde{M}_{0}=2 M_{0}, \quad \tilde{J}=2 J
$$

are sufficient to calculate the parameters of the final disks as functions of the initial parameters. These equations and explicit formulae connecting the gravitational mass $M$, the baryonic mass 


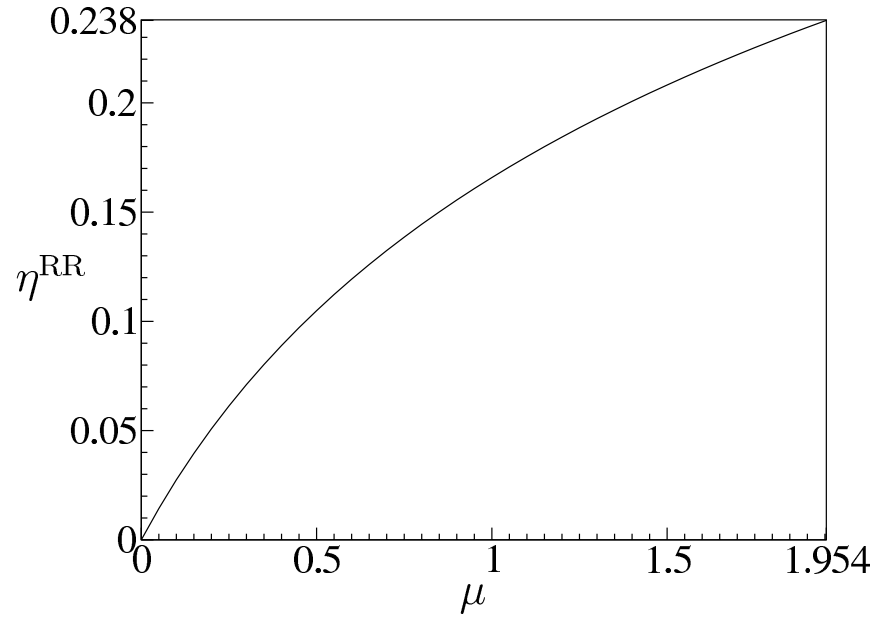

FIG. 4.-Efficiency $\eta_{\mathrm{RR}}$ for the formation of an RR disk from two initial RR disks as a function of the centrifugal parameter $\mu$ of the initial disks. Parameter $\eta^{\mathrm{RR}}$ is an upper limit for the energy loss due to gravitational radiation.

$M_{0}$, and the angular momentum $J$ of the RR disk allowed us to calculate the efficiency $\eta^{\mathrm{RR}}=1-\tilde{M} / 2 M$ as a function of the initial centrifugal parameter $\mu$ (see Fig. 4). It should be emphasized once again that this efficiency measures the total energy loss including friction. Therefore, $\eta$ is only an upper limit for the energy of the gravitational emission. We obtained a maximal value of $\eta_{\max }^{\mathrm{RR}} \approx 23.8 \%$ (Hennig \& Neugebauer 2006).

Furthermore, it turned out that the formation of RR disks from two colliding RR disks is only possible for a rather restricted interval $0<\mu<1.954$. . of the initial centrifugal parameter $\mu$. If $\mu$ exceeds this limit, the collision must lead to other final states, e.g., black holes or black holes surrounded by matter rings.

\subsubsection{Local Conservation Equations}

We now turn to the main goal of this paper and analyze the formation of a disk of dust under the influence of gravitational forces as the only form of interaction. Comparing the resulting differentially rotating disk of dust with the rigidly rotating disk of the same baryonic mass $M_{0}$ and angular momentum $J$ formed from the same initial disks, we may separate gravitational damping due to the emission of gravitational waves from frictional processes in the matter.

We may interpret a disk of dust as a superposition of infinitesimally thin dust rings. Considering the geodesic motion of a single mass element, one can show that for corresponding rings in the two initial disks (see Fig. 5) the baryonic mass and the angular momentum are conserved,

$$
d \tilde{M}_{0}=2 d M, \quad d \tilde{J}=2 d J
$$

i.e., the baryonic masses $d M_{0}$ and the angular momenta $d J$ of the rings with radius $\rho$, taking up the interval $[\rho, \rho+d \rho]$, in each of the two initial disks sum up to $d \tilde{M}_{0}=2 d M_{0}$ and $d \tilde{J}=2 d J$ of the corresponding ring in the final disk (with radius $\tilde{\rho}$, taking up the interval $[\tilde{\rho}, \tilde{\rho}+d \tilde{\rho}]$; see Fig. 5). It should be emphasized that the local conservation laws given by equation (30) would be violated by dissipative processes in the matter or, mathematically speaking, by dissipative terms in the total energy momentum tensor as the source of the Einstein equations during the collision phase. Having reached a final equilibrium configuration (e.g., a rigidly rotating disk of dust), the system "forgets" the dissipative terms and behaves like cold dust with an energy momentum tensor of the form given by equation (26). During the interaction
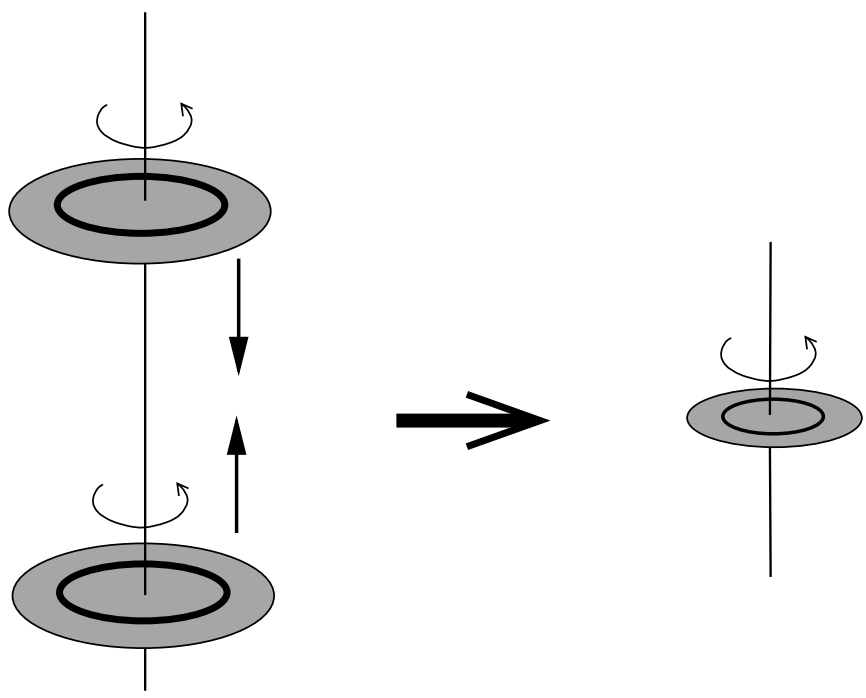

FIG. 5.- Illustration of the local conservation equations. Two corresponding rings of the initial disks merge into a ring in the final disk. The baryonic mass $d M_{0}$ and the angular momentum $d J$ of these rings are conserved.

phase, angular momentum will be transported within the disk by viscous forces and only the total angular momentum (axisymmetry!) and the total baryonic mass are conserved (eq. [29]). The ringwise conservation of baryonic mass and angular momentum given by equation (30) is characteristic for purely gravitational damping processes. They arise from collision processes governed by an energy momentum tensor of dust without dissipative terms. In this case the geodesic motion of the volume elements implies the conservation of baryonic mass and angular momentum in each volume element and therefore implies equation (30).

Equation (30) provides us with a subset of the boundary conditions to be discussed in the next subsection. It will turn out that these conditions, together with conditions resulting from the field equations, determine a unique solution of the Einstein equations describing a final disk with differential rotation (DR disk) as the end product of the collision process.

\subsubsection{Boundary Value Problem for the Final DR Disk}

The line element given by equation (25), which may also be used to describe any axisymmetric and stationary differentially rotating disk, can be reformulated to give

$d \tilde{s}^{2}=e^{2 \tilde{\kappa}}\left(d \tilde{\rho}^{2}+d \tilde{\zeta}^{2}\right)+\tilde{\rho}^{2} e^{-2 \tilde{\nu}}(d \tilde{\varphi}-\tilde{\omega} d \tilde{t})^{2}-e^{2 \tilde{\nu}} d \tilde{t}^{2}$,

where the usage of the functions $\tilde{\kappa}, \tilde{\nu}$, and $\tilde{\omega}($ instead of $\tilde{U}, \tilde{k}$, and $\tilde{a}$ as in eq. [25]) avoids numerical issues with ergospheres (where $\left.e^{2 \tilde{U}}<0\right)$. According to equation (26), the energy momentum tensor is

$$
\tilde{T}^{i j}=\tilde{\varepsilon}(\tilde{\rho}) \delta(\tilde{\zeta}) \tilde{u}^{i} \tilde{u}^{j}
$$

and the four-velocity is again (see eq. [27]) a linear combination of the killing vectors,

$$
\tilde{u}^{i}=e^{-\tilde{V}}\left(\tilde{\xi}^{i}+\tilde{\Omega} \tilde{\eta}^{i}\right),
$$

where $\tilde{V}=\tilde{V}(\tilde{\rho})$ and $\tilde{\Omega}=\tilde{\Omega}(\tilde{\rho})$ are functions of $\tilde{\rho}$ (constancy of $\tilde{V}$ and $\tilde{\Omega}$ defines rigid rotation; see eq. [27]). ${ }^{3}$

\footnotetext{
${ }^{3}$ All quantities of the final DR disk are tilded.
} 
The vacuum field equations for $\tilde{\nu}$ and $\tilde{\omega}$ are (see Bardeen 1973)

$$
\triangle_{1} \tilde{\nu}=\frac{\tilde{\rho}^{2}}{2} e^{-4 \tilde{\nu}}\left(\tilde{\omega}_{, \tilde{\rho}}^{2}+\tilde{\omega}_{, \tilde{\zeta}}^{2}\right), \quad \triangle_{3} \tilde{\omega}=4\left(\tilde{\nu}_{, \tilde{\rho}} \tilde{\omega}_{, \tilde{\rho}}+\tilde{\nu}_{, \tilde{\zeta}} \tilde{\omega}_{, \tilde{\zeta}}\right)
$$

with

$$
\triangle_{n} \equiv \partial_{\tilde{\rho}}^{2}+\partial_{\tilde{\zeta}}^{2}+\frac{n}{\tilde{\rho}} \partial_{\tilde{\rho}}
$$

The matter appears only in the boundary conditions along the $\operatorname{disk}\left(\tilde{\zeta}=0, \tilde{\rho}<\tilde{\rho}_{0}\right)$,

$$
\begin{gathered}
\left.\tilde{\nu}_{, \tilde{\zeta}}\right|_{\tilde{\zeta}=0^{+}}=2 \pi \tilde{\sigma} \frac{1+\tilde{v}^{2}}{1-\tilde{v}^{2}}, \\
\left.\tilde{\omega}_{, \tilde{\zeta}}\right|_{\tilde{\zeta}=0^{+}}=-8 \pi \tilde{\sigma} \frac{\tilde{\Omega}-\tilde{\omega}}{1-\tilde{v}^{2}}, \\
\tilde{\rho}(\tilde{\Omega}-\tilde{\omega})^{2}=\left(1+\tilde{v}^{2}\right) e^{4 \tilde{\nu}} \tilde{\nu}_{, \tilde{\rho}}+\tilde{\rho}^{2}(\tilde{\Omega}-\tilde{\omega}) \tilde{\omega}_{, \tilde{\rho}},
\end{gathered}
$$

where

$$
\tilde{v} \equiv \tilde{\rho} e^{-2 \tilde{\nu}}(\tilde{\Omega}-\tilde{\omega}), \quad \tilde{\sigma} \equiv \tilde{\varepsilon} e^{2 \tilde{\kappa}} .
$$

Thus, we have to deal with a boundary value problem for Einstein's vacuum equations.

As already mentioned, the local conservation equations given by equation (30) of the previous subsection lead to additional boundary conditions along the disk. From $d \tilde{M}_{0}=2 d M_{0}$ with $d M_{0}=2 \pi \sigma e^{-V_{0}} \rho d \rho$ and $d \tilde{M}_{0}=2 \pi \tilde{\sigma} e^{-\tilde{V}} \tilde{\rho} d \tilde{\rho}$ we obtain

$$
\tilde{\sigma}=2 \sigma \frac{\rho e^{V_{0}}}{\tilde{\rho} e^{\tilde{V}}} \frac{d \rho}{d \tilde{\rho}} .
$$

Likewise, $d \tilde{J}=2 d J$ with $\quad d J=2 \pi \sigma e^{-V_{0}} u^{i} \eta_{i} \rho d \rho, d \tilde{J}=$ $2 \pi \tilde{\sigma} e^{-\tilde{V}} \tilde{u}^{i} \tilde{\eta}_{i} \tilde{\rho} d \tilde{\rho}, u^{i} \eta_{i}=\rho v e^{-V_{0}}$, and $\tilde{u}^{i} \tilde{\eta}_{i}=\tilde{\rho} \tilde{v} e^{-\tilde{V}}$ leads to

$$
\tilde{\rho} \tilde{v} e^{-\tilde{V}}=\rho v e^{-V_{0}} .
$$

Equations (40) and (41) relate the boundary values of the DR disk to the analytically known mass density and the metric potentials of the RR disk (see the Appendix). The function $\tilde{V}(\tilde{\rho})$ can be calculated from $\tilde{u}^{i} \tilde{u}_{i}=-1$,

$$
e^{2 \tilde{V}}=\left(1-\tilde{v}^{2}\right) e^{2 \tilde{\nu}}
$$

The remaining boundary conditions describe the behavior at infinity, where the metric approaches the flat Minkowski metric,

$$
\tilde{\kappa}=\tilde{\nu}=\tilde{\omega}=0,
$$

and in the plane $\tilde{\zeta}=0$ outside the disk $\left(\tilde{\rho}>\tilde{\rho}_{0}\right)$, where equations (36) and (37) lead to vanishing normal derivatives,

$$
\left.\tilde{\nu}_{, \tilde{\zeta}}\right|_{\tilde{\zeta}=0^{+}}=0,\left.\quad \tilde{\omega}_{, \tilde{\zeta}}\right|_{\tilde{\zeta}=0^{+}}=0 .
$$

In addition, we have to ensure regularity along the axis of symmetry $\tilde{\rho}=0$.

Equations (34), (36)-(38), (40), and (41) form a complete set of equations to determine the unknown functions uniquely: there are two two-dimensional functions, $\tilde{\nu}(\tilde{\rho}, \tilde{\zeta})$ and $\tilde{\omega}(\tilde{\rho}, \tilde{\zeta})$, which

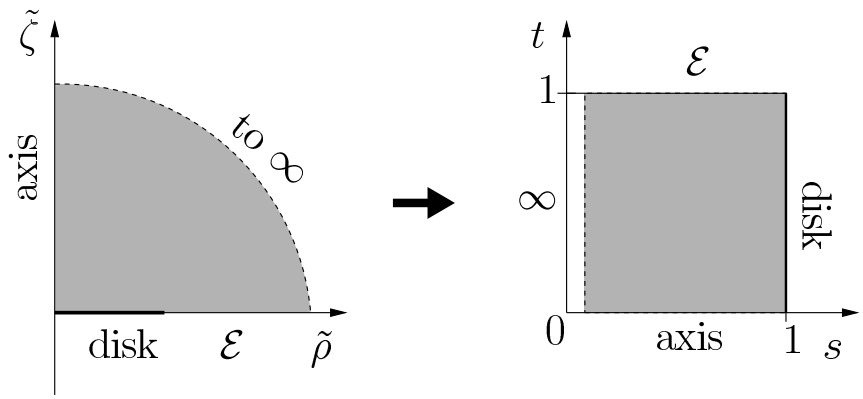

FIG. 6.-Coordinate transformation given by eqs. (45) and (46) mapping the part $\tilde{\zeta} \geq 0$ of the $(\tilde{\rho}, \tilde{\zeta})$-plane to a unit square in the $(s, t)$-plane. $\mathcal{E}$ denotes the equatorial plane outside the disk, $\tilde{\zeta}=0, \tilde{\rho}>\tilde{\rho}_{0}$.

have to satisfy the two elliptic partial differential equations given by equation (34) with the boundary conditions given by equations (36) and (37), and three additional one-dimensional functions in the disk, $\tilde{\Omega}(\tilde{\rho}), \tilde{\sigma}(\tilde{\rho})$, and $\rho(\tilde{\rho})$, which have to obey the three boundary conditions given by equations (38), (40), and (41). (The metric function $\tilde{\kappa}$ can be calculated by a line integral afterward but is not needed for the computation of the efficiency $\eta$ in our collision scenario.)

\subsubsection{Numerical Method}

In order to prepare numerical investigations, we map the region $0 \leq \tilde{\rho} \leq \infty, 0 \leq \tilde{\zeta} \leq \infty$ to a unit square, thus reaching a compactification of infinity (see Fig. 6). (Due to the reflection symmetry with respect to the plane $\tilde{\zeta}=0$, we can restrict ourselves to the region $\tilde{\zeta} \geq 0$.) To do this, we introduce in a first step elliptical coordinates

$$
\tilde{\rho}=\sqrt{\left(1+\xi^{2}\right)\left(1-\eta^{2}\right)}, \quad \tilde{\zeta}=\xi \eta, \quad \xi \in[0, \infty], \quad \eta \in[0,1]
$$

(without loss of generality, we may choose units where $\tilde{\rho}_{0}=1$ ). In a second step we stretch the coordinates by the transformation

$$
\xi=\cot \left(\frac{\pi}{2} s\right), \quad \eta=\sqrt{1-t}, \quad s \in[0,1], \quad t \in[0,1] .
$$

The coordinates $s$ and $t$ form a unit square with the following boundaries:

$$
\begin{array}{ll}
s=0: & \infty, \\
s=1: & \text { disk, } \tilde{\rho} \leq 1, \tilde{\zeta}=0, \\
t=0: & \text { axis of symmetry, } \tilde{\rho}=0, \\
t=1: & \text { disk plane } \mathcal{E} \text { outside the matter, } \tilde{\rho}>1, \tilde{\zeta}=0 .
\end{array}
$$

The unknown functions in the boundary value problem are analytic functions in this square (as is known for the case of Maclaurin disks or the RR disks). Hence, it is convenient to use spectral methods for the numerical solution of the boundary value problem. We expand the unknown potentials in terms of Chebyshev polynomials $T_{j}$ to a predetermined order in the form

$$
\begin{aligned}
& f(s, t)=\sum_{j, k} c_{j k} T_{j}(2 s-1) T_{k}(2 t-1), \\
& f(t)=\sum_{k} c_{k} T_{k}(2 t-1) \quad \text { (boundary) }
\end{aligned}
$$




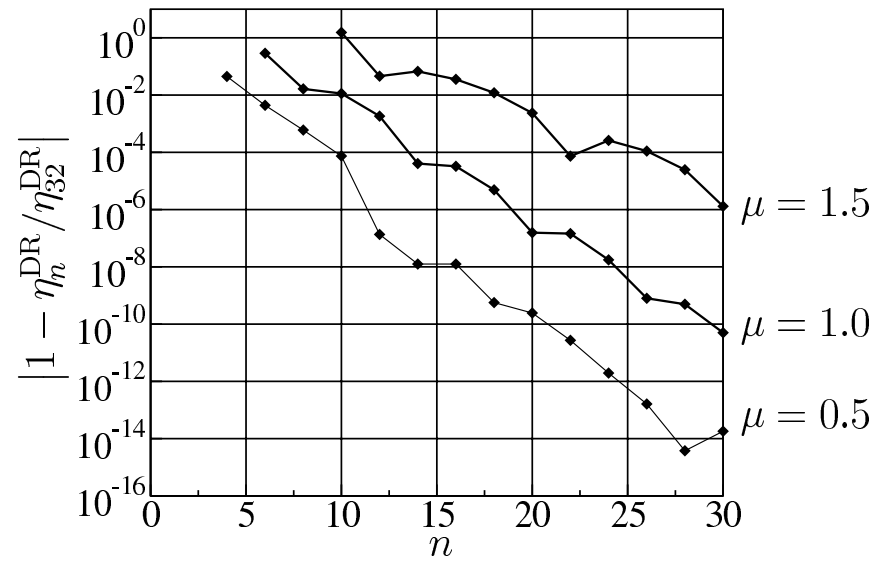

FIG. 7.-Convergence properties of the numerical code for the example $\eta^{\text {DR }}$. The values of the efficiency $\eta^{\mathrm{DR}}$ for different orders $n_{s}=n_{t}=n$ of the Chebyshev expansion are related to the order $n=32$. The plot shows $\left|1-\eta_{n}^{\mathrm{DR}} / \eta_{32}^{\mathrm{DR}}\right|$ as a function of $n$.

and formulate the Einstein equations at the extrema of the Chebyshev polynomials. This leads to an algebraic system of equations for the Chebyshev coefficients (or, alternatively, for the values of the potentials at these points) that can be solved with the Newton-Raphson method. The iteration starts with an initial "guessed" solution (for example, the Newtonian approximation; see $\S 3.2 .6$ ).

The calculations show a decreasing accuracy of the numerical solution for increasingly large values of the initial parameter $\mu$. The reason are large gradients of the metric potentials for strong relativistic DR disks that make the Chebyshev approximation more costly. To reach a better convergence, we perform an additional coordinate transformation

$$
s=\frac{\sinh \delta \tilde{s}}{\sinh \delta}
$$

introducing a new coordinate $\tilde{s}$, where $\delta$ is a suitably chosen parameter. As shown in Ansorg \& Petroff (2005), this transformation smooths the gradients of the metric functions. The convergence is illustrated in Figure 7.

\subsubsection{Results}

Using this numerical algorithm, we are able to solve the boundary value problem for the final DR disk. In particular, we could calculate, for each value of the initial parameter $\mu$, all metric coefficients of this final disk. However, we restrict ourselves to the discussion of the relations between the initial and final parameters and the efficiency of the collision process. Especially, we compare the final DR disk with an RR disk having the same baryonic mass and angular momentum. The point made here is that such a rigidly rotating disk represents the state of "thermodynamic equilibrium" for disks of dust as the end point of their thermodynamic evolution. As sketched in Figure 8, there are at least two possibilities for the formation of this final RR disk: the direct process (scenario A) including friction from the beginning or the equivalent thermodynamic process (scenario B) where, in a first step, a differentially rotating disk is formed (by gravitational damping alone, no friction) and, in a second step, the angular velocity becomes constant (due to friction). Note that baryonic mass and angular momentum are conserved in both processes. By comparing scenarios $\mathrm{A}$ and $\mathrm{B}$, we may extract the contribution of friction in scenario A.
(A)
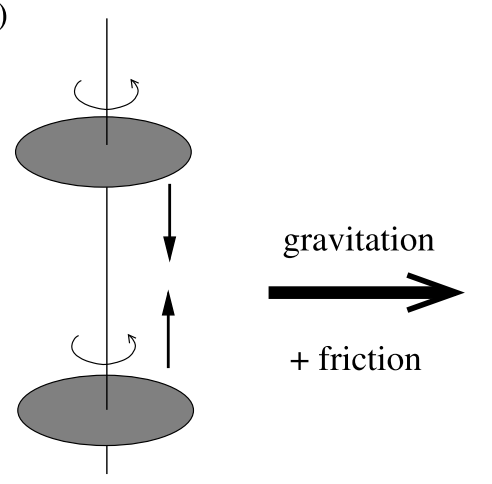

+ friction

(B)

$$
\text { RR disks }
$$

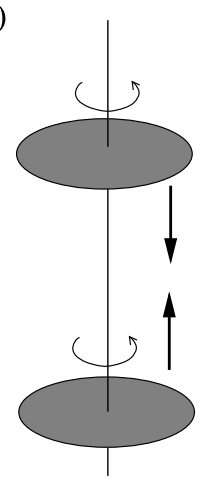

RR disks

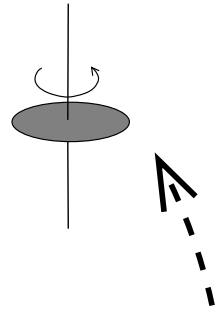

RR disk

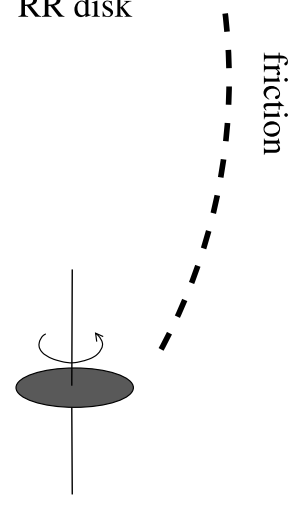

DR disk

FIG. 8.- Two models for disk collisions: (A) Under the influence of a small amount of friction, RR disks merge again into an RR disk. This scenario was discussed in Hennig \& Neugebauer (2006); see $\S 3.2 .1$. (B) In the absence of friction, the same RR disks merge into a DR disk. Allowing for friction afterward, the system would again arrive at the RR disk of scenario A after a sufficiently long time.

In the following discussion, tilded quantities, as before, belong to the final DR disk of scenario B in Figure 8, a superscript "RR" denotes quantities of the final RR disk in scenario A, and the centrifugal parameter $\mu$ without any additions characterizes the initial RR disks.

The rotation curve of the final DR disk, i.e., its (normalized) angular velocity $\tilde{\Omega} \tilde{\rho}_{0}$ as a function of the (normalized) radius $\tilde{\rho} / \tilde{\rho}_{0}$, is shown in the top panels of Figure 9 . For small parameters $\mu$ (post-Newtonian regime) the function $\tilde{\Omega} \tilde{\rho}_{0}$ is almost constant (top left panel). Interestingly, strongly relativistic disks $(\mu \gtrsim 1.5)$ show the same property (top right panel). Moreover, $\tilde{\Omega} \tilde{\rho}_{0}$ tends to zero in the ultrarelativistic limit in analogy to the relation $\Omega^{\mathrm{RR}} \rho_{0}^{\mathrm{RR}} \rightarrow 0$, which holds for RR disks in the ultrarelativistic limit $\mu^{\mathrm{RR}} \rightarrow 4.62966 \ldots$

The "centrifugal parameter" $\tilde{\mu}=2 \tilde{\Omega}^{2} \tilde{\rho}_{0}^{2} e^{-\tilde{V}}=\mu(\tilde{\rho})$ is shown in the middle left panel of Figure 9. Like the angular velocity, $\tilde{\mu}$ is almost constant for small $\mu$. For strongly relativistic DR disks, $\tilde{\mu}$ in the center of the disk exceeds the limit $\mu_{\max }^{\mathrm{RR}}=4.62966 \ldots$ of RR disks.

The middle right panel of Figure 9 shows the quantity $\tilde{\Omega} \tilde{M}$ as a function of $\rho / \rho_{0}$. For strongly relativistic DR disks $\tilde{\Omega} \tilde{M}$ becomes constant and approaches the limit 0.5 . On the other hand, this is a characteristic value for extreme Kerr black holes where $\Omega_{H} M_{\mathrm{BH}}=0.5\left(\Omega_{H}\right.$ : angular velocity of the horizon; $M_{\mathrm{BH}}$ : black hole mass). Indeed, one can show that there is a phase transition between RR disks and Kerr black holes (Bardeen \& Wagoner 

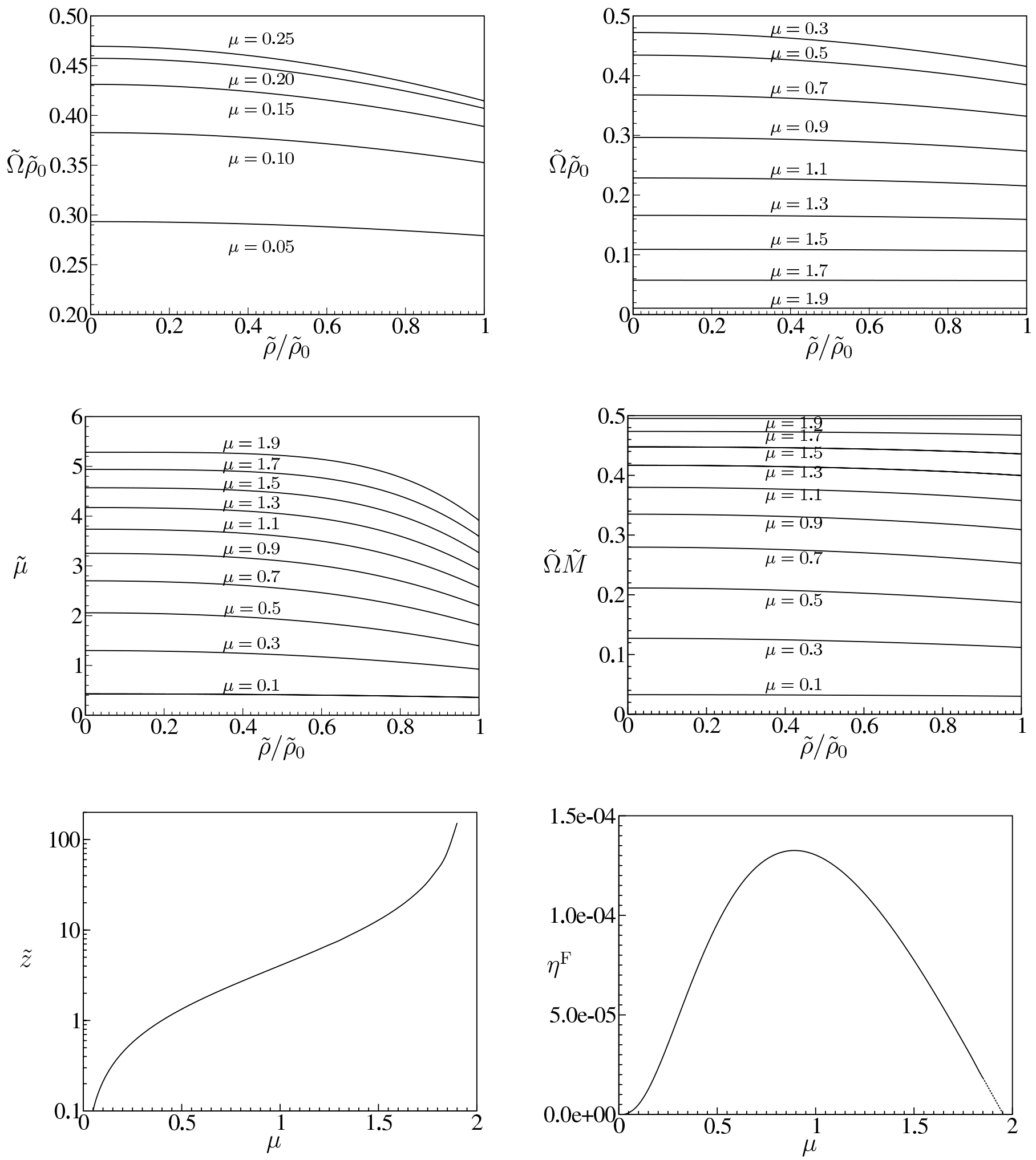

FIG. 9.-Parameter relations for the collision of RR disks. We performed numerical calculations for values of the initial centrifugal parameter $\mu$ in the interval [0, 1.9]. The dotted part of the line in the bottom right panel is an extrapolation for larger $\mu$. This extrapolation and the rapidly growing redshift in the bottom left panel indicate that the initial parameter $\mu$ in scenario B is limited (approximately, or perhaps even exactly) to the same interval as in scenario A, $0<\mu<1.954$. . (see $\S 3.2 .1$ ).

1971; Neugebauer \& Meinel 1994). This inspires the conjecture that the DR disk exhibits the same phase transition. There is no obstacle for a (numerical) proof of this assumption in principle. To extend our present code to study the parametric collapse of the DR disk including the formation of a horizon, we would have to follow the ideas of Bardeen \& Wagoner (1971), who analyzed this problem for the RR disks. However, such investigations are outside the scope of this paper.

The bottom left panel of Figure 9 shows the redshift $\tilde{z}$ for a photon emitted from the disk center as a function of the initial 
centrifugal parameter $\mu$. For increasing values of $\mu$ (relativistic DR disks) $\tilde{z}$ grows rapidly.

An important result is the efficiency $\eta^{\mathrm{DR}}$ of the formation of DR disks that measures the amount of energy converted into gravitational radiation. The difference $\eta^{F}=\eta^{\mathrm{RR}}-\eta^{\mathrm{DR}}$, as shown in the bottom right panel of Figure 9, compares this value with the efficiency $\eta^{\mathrm{RR}}$ of the RR disk-forming process as sketched in scenario A of Figure 8 . Thereby, $\eta^{F}$ is the part of energy lost due to friction during the formation of a final RR disk. We find $\eta^{F}=$ $\eta^{\mathrm{RR}}-\eta^{\mathrm{DR}}<1.5 \times 10^{-4}$, i.e., the contribution of friction is extremely small, $\eta^{F} \ll \eta^{\mathrm{RR}}$, such that the gravitational radiation dominates the collision process A.

\subsubsection{Analytical Treatment of the Newtonian Limit}

Our numerical investigations have shown that the angular velocity of the final DR disk becomes closer and closer to a constant over the whole range of $\tilde{\rho} / \tilde{\rho}_{0}$ as the centrifugal parameter $\mu$ tends to zero (see Fig. 9, top left panel). This leads one to suspect that a final disk with a strictly constant angular velocity will solve the boundary value problem as discussed in $\S 3.2 .3$ in Newtonian theory. Interestingly, we can treat this problem analytically. This is demonstrated here. Strictly speaking, there is no gravitational radiation in Newton's theory. However, this Newtonian boundary value problem can be seen as the limit of a sequence of relativistic collisions with decreasing $\mu$, all reaching a final equilibrium state due to gravitational emission. Moreover, the Newtonian solution can be used as a starting point for the iterative calculation of the final relativistic DR disk.

Since the Newtonian limit of the RR disk is the Maclaurin disk, we have to study the collision of two identical Maclaurin disks using the local conservation laws given by equation (30). The Newtonian potential $\tilde{U}$ of the final disk is a solution of the Poisson equation

$$
\triangle \tilde{U}=4 \pi \tilde{\sigma} \delta(\zeta)
$$

with the boundary condition

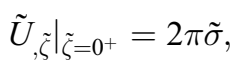

where $\tilde{\sigma}=\tilde{\sigma}(\tilde{\rho})$ is the surface mass density of the final disk. With $d M=2 \pi \sigma d \rho$ and $d J=\Omega \rho^{2} d M$, equation (30) leads to the additional boundary conditions

$$
\tilde{\sigma}(\tilde{\rho})=2 \sigma(\rho) \frac{\rho}{\tilde{\rho}} \frac{d \rho}{d \tilde{\rho}}, \quad \tilde{\Omega}(\tilde{\rho}) \tilde{\rho}^{2}=\Omega_{0} \rho^{2} .
$$

The initial surface mass density of the Maclaurin disk is

$$
\sigma(\rho)=\frac{3 M}{2 \pi \rho_{0}^{2}} \sqrt{1-\frac{\rho^{2}}{\rho_{0}^{2}}}
$$

and the initial constant angular velocity $\Omega_{0}$ is related to the initial mass by

$$
\Omega_{0}^{2}=\frac{3 \pi M}{4 \rho_{0}^{3}} .
$$

Using these relations, together with the Euler equation

$$
\left.\tilde{U}_{, \tilde{\rho}}\right|_{\tilde{\zeta}=0}=\tilde{\Omega}^{2}(\tilde{\rho}) \tilde{\rho},
$$

we find that a (rigidly rotating) Maclaurin disk with the parameters

\begin{tabular}{|c|c|}
\hline Colliding Objects & $\begin{array}{l}\eta_{\max } \\
(\%)\end{array}$ \\
\hline 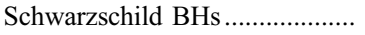 & 29.3 \\
\hline 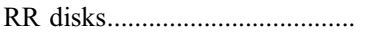 & 23.8 \\
\hline Schwarzschild stars ...................... & 19.7 \\
\hline 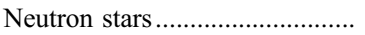 & 2.3 \\
\hline
\end{tabular}

$$
\tilde{\Omega}=4 \Omega_{0}, \quad \tilde{\rho}_{0}=\frac{1}{2} \rho_{0}
$$

TABLE 1

UPPER LiMiTS FOR THE EFFICIENCY $\eta$ of Different Collision Processes

Notes.-Upper limits for the efficiency $\eta$ of different collision processes, including the Hawking \& Ellis (1973) limit for the collision of two spherically symmetric black holes. According to the bottom right panel of Fig. 9 $\left[\eta^{F}(1.954 \ldots)=0\right]$, the two efficiencies $\eta^{\text {RR }}$ and $\eta^{\mathrm{DR}}$ coincide with a maximum value $\eta_{\max }^{\mathrm{RR}}=\eta_{\max }^{\mathrm{DR}} \approx 23.8 \%$.

indeed solves the boundary value problem given by equations (50)-(52).

\section{DISCUSSION}

In this paper we have performed the analysis of collision processes in the spirit of equilibrium thermodynamics. Avoiding the solution of the full dynamical problem, we compared initial and final equilibrium configurations to obtain a "rough" picture of these processes. In this way we were able to calculate the energy loss by the emission of gravitational waves and to find conditions ("parameter relations") for the formation of final stars and disks.

The application of this method to collisions of perfect fluid stars and collisions of rigidly rotating disks of dust leads to restrictions of the initial parameters. It turned out that the formation of final stars/disks from stars/disks is only possible for a subset of the parameter space of the initial objects. Otherwise, the collision of spheres and disks would lead to other final states, e.g., to black holes.

Our main result is the numerical solution of the Einstein equations for the differentially rotating (DR) disk formed by the collision of two identical rigidly rotating ( $R R$ ) disks with parallel angular momenta. We calculated the characteristic quantities of the final DR disk, as for example the rotation curve $\tilde{\Omega}(\tilde{\rho})$, as it depends on the centrifugal parameter $\mu$ of the initial RR disks. It turned out that the angular velocity $\tilde{\Omega}$ is almost constant (as shown in $\S 3.2 .6$, it is strictly constant in the Newtonian limit). Therefore, the simplified model of the formation of an RR disk from the collision of two RR disks as presented in Hennig \& Neugebauer (2006), which has to allow frictional processes to reach constant angular velocity, turns out to be a good approximation to our present purely gravitational (frictionless) model (scenario B).

For each of the studied collision scenarios, we calculated an upper limit for the energy of the emitted gravitational waves. A summary of the maximal efficiencies is given in Table 1 . The value $\eta_{\max } \approx 2.3 \%$ for the collision of neutron stars is relatively small compared to the other examples. The reason is the restricted equation of state (completely degenerate ideal Fermi gas) that does not allow for strongly relativistic stars.

We would like to thank David Petroff for many valuable discussions. This work was supported by the Deutsche Forschungsgemeinschaft (DFG) through the SFB/TR7 "Gravitationswellenastronomie." 


\section{APPENDIX}

\section{POTENTIALS OF THE RIGIDLY ROTATING DISK OF DUST}

For the numerical calculation of the DR disk that is formed by the collision of two RR disks we need some formulae for quantities of the RR disk of dust.

The coefficient $V_{0}$ in the four-velocity given by equation (27) as a function of the parameter $\mu$ can be calculated from a very rapidly converging series (see Kleinwächter 1995),

$$
\begin{aligned}
\operatorname{coth} \frac{V_{0}}{2}= & -\frac{4}{\mu}+0.0294938052100425142 \mu+5.4681333461446 \times 10^{-6} \mu^{3} \\
& -1.07467432587 \times 10^{-9} \mu^{5}+2.1127368 \times 10^{-13} \mu^{7}-4.154 \times 10^{-17} \mu^{9}+\mathcal{O}\left(\mu^{11}\right) .
\end{aligned}
$$

The disk values $\left(\zeta=0, \rho \leq \rho_{0}\right)$ of the metric functions $U$ and $a$ and the mass density $\sigma$ are given by the equations

$$
\begin{gathered}
e^{2 U}=e^{2 V_{0}(\hat{\mu})}-\frac{\mu \rho^{2}}{2 \rho_{0}^{2}}, \\
\left(1+\Omega_{0} a\right) e^{2 U}=e^{V_{0}(\mu)} e^{V_{0}(\hat{\mu})}, \\
\sigma=-\frac{\Omega_{0}}{2 \pi e^{V_{0}(\mu)}} \frac{b_{0}^{\prime}(\hat{\mu})}{e^{V_{0} \hat{\mu}}},
\end{gathered}
$$

with

$$
\Omega_{0}=\sqrt{\frac{\mu}{2}} \frac{e^{V_{0}}}{\rho_{0}}, \quad b_{0}=-\sqrt{1-e^{4 V_{0}}-4 \Omega_{0}^{2} \rho_{0}^{2}}
$$

(see Neugebauer \& Meinel 1994). The notation $V_{0}(\hat{\mu}), b_{0}^{\prime}(\hat{\mu})$ indicates that the argument $\mu$ in the parameter functions $V_{0}(\mu)$ and $b_{0}^{\prime}(\mu)$ has to be replaced by $\hat{\mu}=\left(1-\rho^{2} / \rho_{0}^{2}\right) \mu$. Here $b_{0}^{\prime}(\hat{\mu})$ means $d b_{0}(\hat{\mu}) / d \hat{\mu}$.

\section{REFERENCES}

Anninos, P., \& Brandt, S. 1998, Phys. Rev. Lett., 81, 508

Anninos, P., Hobill, D., Seidel, E., Smarr, L., \& Suen, W.-M. 1993, Phys. Rev.

Lett., 71, 2851

1995, Phys. Rev. D, 52, 2044

Ansorg, M. 2001, Gen. Relativ. Gravitation, 33, 309

Ansorg, M., \& Meinel, R. 2000, Gen. Relativ. Gravitation, 32, 1365

Ansorg, M., \& Petroff, D. 2005, Phys. Rev. D, 72, 024019

Bardeen, J. M. 1973, in Black Holes, ed. C. DeWitt-Morette \& B. S. DeWitt (New York: Gordon \& Breach), 215

Bardeen, J. M., \& Wagoner, R. V. 1969, ApJ, 158, L65

. 1971, ApJ, 167, 359

Beig, R., \& Simon, W. 1992, Commun. Math. Phys., 144, 373

Eppley, K. 1975, Ph.D. thesis, Princeton Univ.

Fiske, D. R., Baker, J. G., van Meter, R. R., Choi, \& D.-I., \& Centrella, J. M. 2005, Phys. Rev. D, 71, 104036

Hawking, S. W., \& Ellis, G. F. R. 1973, The Large Scale Structure of SpaceTime (Cambridge: Cambridge Univ. Press)

Hennig, J., \& Neugebauer, G. 2006, Phys. Rev. D, 74, 064025

Kleinwächter, A. 1995, Ph.D. thesis, Friedrich-Schiller-Univ. Jena
Lindblom, L., \& Masood-Ul-Alam, A. K. M. 1994, Commun. Math. Phys., 162,123

Löffler, F., Rezzolla, L., \& Ansorg, M. 2006, Phys. Rev. D, 74, 104018

Masood-Ul-Alam, A. K. M. 2007, Gen. Relativ. Gravitation, 39, 55

Misner, C. W., Thorne, K. S., \& Wheeler, J. A. 2002, Gravitation (New York: Freeman)

Neugebauer, G., Kleinwächter, A., \& Meinel, R. 1996, Helvetica Phys. Acta, 69,472

Neugebauer, G., \& Meinel, R. 1993, ApJ, 414, L97

_. 1994, Phys. Rev. Lett., 73, 2166 1995, Phys. Rev. Lett., 75, 3046

Shapiro, S. L., \& Teukolsky, S. A. 1983, Black Holes, White Dwarfs, and Neutron Stars: The Physics of Compact Objects (New York: Wiley)

Smarr, L., Čadež, A., DeWitt, B., \& Eppley, K. 1976, Phys. Rev. D, 14, 2443 Sperhake, U. 2006, Phys. Rev. D, submitted (gr-qc/0606079)

Sperhake, U., Kelly, B., Laguna, P., Smith, K. L., \& Schnetter, E. 2005, Phys. Rev. D, 71, 124042

Zlochower, Y., Baker, J. G., Campanelli, M., \& Lousto, C. O. 2005, Phys. Rev. D, 72,024021 